\title{
Fragen zwischen Sozial- und Rechtswissenschaften bei familienbezogener Forschung - aus rechtswissenschaftlicher Sicht
}

\section{Mutual questions regarding family-related research in social sciences and jurisprudence - Questions posed from the viewpoint of jurisprudence}

\begin{abstract}
Zusammenfassung
Die Zusammenarbeit von Sozial- und Rechtswissenschaften bei familienbezogener Forschung erscheint als außerordentlich wichtig, weil diese Disziplinen vielfach aufeinander Bezug nehmen. Gemeinsam ist ihnen, dass sie ein Auseinanderfallen von Rechtsnormen und gesellschaftlicher Realität für möglich und wissenschaftlich darstellbar halten und daraus rechtspolitische Forderungen ableiten. Unterschiedliche Aufgabenstellungen und Sichtweisen sowie unterschiedliche Terminologie und Methodik bringen indes die Gefahr von Missverständnissen hervor, die nur durch gegenseitige Verständigung und Zusammenarbeit offengelegt werden kann. Der Beitrag erläutert diesen Befund anhand einiger familiärer Konstellationen wie Ehe, nichteheliche Lebensgemeinschaft, Ehescheidung und Paartrennung, rechtliche und soziale Elternschaft, alleinerziehende Eltern, Stieffamilie, sozialfamiliäre Beziehung. Den Schluss bildet ein Plädoyer aus Sicht der Rechtswissenschaft für die Intensivierung transdisziplinärer Forschung.
\end{abstract}

Schlagwörter: Ehe, nichteheliche Lebensgemeinschaft, Kohabitation, De-Institutionalisierung, Individualisierung, Solidarität, Ehescheidung, Trennung, Verschuldensprinzip, Zerrüttungsprinzip, Scheidungsfolgen, Unterhalt, Rententeilung (,Splitting“), Elternfamilie, Ein-Elternfamilie, Alleinerziehende, soziale Elternschaft, sozial-familiäre Beziehung, Stieffamilie, Patchworkfamilie.

\begin{abstract}
Since social sciences and jurisprudence refer to each other in family-related research in many ways, it appears to be extremely important that both disciplines collaborate. They both have in common that they consider it possible that legal norms and societal realities diverge and that this division can be delineated scientifically. From this divergence, they deduce calls for consequences in legal policy. Diverging tasks and viewpoints as well as differing terms and methods may lead to misconceptions that, in turn, can only be identified by mutual comprehension and collaboration. In the contribution presented here, these findings shall be exemplified by several family constellations, such as marriage, civil union, separation and divorce, legal and social parenthood, single parents, stepfamily, socio-family relationship. In the conclusion, there shall be a plea from the viewpoint of jurisprudence - for an intensification of transdisciplinary research.
\end{abstract}

Key words: marriage, civil union, cohabitation, de-institionalization, individualization, solidarity, divorce, separation, legal principle of fault for divorce, legal principle of marital breakdown, alimony, pension sharing orders ("splitting"), singleparent family, single parents, social parenthood, socio-family relationship, stepfamily, patchwork family. 


\section{Zur Begegnung der Disziplinen}

Ersten Anlass zur Beschäftigung mit dem Thema gab mir ein Symposium, das im November 2010 in Bamberg stattfand und mich auch noch nachträglich begeistert. Knapp zwanzig Wissenschaftlerinnen und Wissenschaftler aus der Soziologie, Psychologie und der Jurisprudenz kamen für drei Tage zusammen, um das Phänomen der „Pluralisierung von Elternschaft und Kindschaft" in den wissenschaftlichen Blick zu nehmen. Der sehr furchtbare Gedankenaustausch offenbarte auch gewisse Probleme, welche die beteiligten Wissenschaften mit der gegenseitigen Verständigung haben können. Ich habe damals einige Gedanken dazu niedergelegt, die ich hier weiterverfolgen will.

Die Forderung nach einer Zusammenarbeit von Sozialwissenschaften auf der einen, Rechtswissenschaft und Rechtspolitik auf der anderen Seite ist alt und wohl begründet. Vom Recht aus gesehen lässt sich die Situation wie folgt beschreiben. Rechtliche Normen haben den Sinn, das Verhalten der Menschen zu leiten mit dem Ziel, Gerechtigkeit unter ihnen zu schaffen und gleichzeitig für die gesamte Gesellschaft ein funktionierendes Regelwerk zur Verfügung zu stellen. Das Recht zielt auf Realisierung, nicht nur im Einzelfall bei Gericht, sondern als eine die Gesellschaft leitende Regel. Weil das so ist, machen sich die rechtspolitischen Akteure (Gesetzgebung, Rechtsprechung, Wissenschaft) notwendigerweise ein Bild von der gesellschaftlichen Realität.

Nun gibt es Wissenschaften, die den mit dem Recht befassten Professionen helfen können, sich ein zutreffendes Bild von der Wirklichkeit zu machen, das sind im Bereich der Familie vor allem die Soziologie und die Psychologie. So liegt es nahe, dass Rechtspolitik und Rechtswissenschaft die Erkenntnisse dieser Wissenschaften für ihre Realitätswahrnehmung zu Rate ziehen.

Die Sozialwissenschaften ihrerseits - ich beschränke mich im Folgenden auf die Soziologie - nehmen das reale Verhalten der Menschen und ihrer Gruppen in den Blick, deuten es, stellen es in einen theoretischen Rahmen; sie wissen dabei, dass dieses Verhalten auch durch Rechtsregeln und Rechtsvorstellungen beeinflusst wird. Für die Soziologie ergibt sich dann die Frage, welche Rolle der „rechtliche Hintergrund“ in ihren Analysen spielen kann. Wie wirken Gesetzgebung und Rechtsprechung auf das Verhalten der Gesellschaftsmitglieder?

Dabei ist es offenbar ist es nicht so, dass das Recht unmittelbar die Realität bestimmt: Obwohl der Diebstahl verboten ist, wird massenhaft gestohlen. Der „Ist-Zustand“ einer Gesellschaft entspricht nie dem rechtlichen „Soll-Zustand“. Doch bedeutet das möglicherweise nicht die Wirkungslosigkeit des Rechts. Man könnte die Hypothese bilden: Die Rechtordnung bildet eine latente Größe, die keineswegs die Realität widerspiegelt, ohne welche aber die Realität nicht so wäre, wie sie ist. In diesem - eingeschränkten - Sinne könnte man das Recht als Teil der Realität selbst („Wirklichkeit“ im Schopenhauer'schen Sinne) begreifen.

So gesehen müssten sich Sozial- und Rechtswissenschaften eigentlich täglich begegnen. Das das nicht so häufig ist, wie es sein könnte, mag unter anderem auch mit Problemen zusammenhängen, die einerseits das Recht bei der Vorstellung von gesellschaftlicher Wirklichkeit, andererseits die Soziologie beim Umgang mit dem Recht hat. 
- Die Juristen denken in den Bahnen ihres Regelsystems. Auch wenn sie sich klarmachen, dass die Normen zunächst einmal bloße Abstrakta sind, kommen sie von ihrer Rechtsbegrifflichkeit schwer los. Die Rechtsbegriffe wirken wie eine Brille, die das Sichtfeld begrenzt.

- Die Soziologen haben mit der Schwierigkeit zu kämpfen, dass sie einerseits den rechtlichen Kontext der gesellschaftlichen Vorgänge zur Kenntnis nehmen, andererseits natürlich wissen, dass das nicht die Realität ist. Die Folge ist - nach meiner subjektiven Wahrnehmung - ein ambivalentes Verhältnis der Sozialwissenschaften zum Recht. Der „rechtliche Hintergrund“ wird möglichst am Rande belassen, oder überhaupt ausgeblendet.

Sind Soziologen und Juristen in bestimmtem Grade Gefangene ihres professionellen Blicks, so trennt sie zudem die Terminologie. Es ist nicht nur so, dass dieselben Termini Unterschiedliches bedeuten können („Kind“), sondern dass vielfach Begriffe verwendet werden, die in der jeweils anderen Fachsprache kein Pendant haben, also zwischen den Wissenschaften nicht übersetzbar sind, so die noch zu besprechende „sozial-familiäre Beziehung“" auf der juristischen, die „soziale Elternschaft“ auf der soziologischen Seite.

Ich möchte die Probleme anhand einiger Sachthemen kurz beleuchten, natürlich aus dem juristischen Blick - einen anderen habe ich nicht zu bieten.

\section{Ehe und nichteheliche Lebensgemeinschaft}

Zunächst zum Thema „Ehe und nichteheliches Zusammenleben“. Hier stehen im Mittelpunkt der sozialwissenschaftlichen Beschreibungen und Deutungen vor allem der Rückgang der Eheschließungen, die Zunahme der Scheidungsraten, die Vermehrung von nichtehelichen Lebensgemeinschaften und die Zunahme der Single-Haushalte.

Man schließt aus diesen Beobachtungen, dass die gesellschaftliche Bedeutung der Ehe rückläufig ist und sucht dafür Erklärungen, die zwischen Tendenzbeschreibung und Ursachenverknüpfung angesiedelt sind. Es ist die Rede von „Individualisierung“"1, „DeInstitutionalisierung “', ,Pluralisierung und Differenzierung “3 Liebe und Ehe“, von Entkoppelung von „Ehe und Elternschaft“, 4 von „Wertewandel von

1 U. Beck/E. Beck-Gernsheim, Das ganz normale Chaos der Liebe, Frankfurt a.M. 1990, S. 11f.; dazu F.-X. Kaufmann, Zukunft der Familie im vereinten Deutschland. Gesellschaftliche und politische Bedingungen, München 1995, S. 102; L. A. Vaskovics/M. Rupp/B. Hofmann, Lebensläufe in der Moderne. Nichteheliche Lebensgemeinschaften. Eine soziologische Längsschnittstudie, Opladen 1997, S. 32; G. Burkart, Familiensoziologie, Konstanz 2008, S. 237 (zentrales Merkmal), kritisch; P. B. Hill/J. Kopp, Familiensoziologie. Grundlagen und theoretische Perspektiven, 5. Aufl. Wiesbaden 2013, S. 144ff., S. $261 \mathrm{ff}$.

2 Kaufmann (wie Fn.1), S. 165.

3 M. Rupp, Die nichteheliche Lebensgemeinschaft als Bindungsphase. Paarkonstellationen und Bindungsprozesse, Hamburg 1999, 43. Kritisch zur Pluralisierungsthese Hill/Kopp (Fn. 1), S. 255; Burkart (wie Fn. 1), S. 34.

4 Kaufmann (Fn. 1), S. 98f. 
Pflicht- und Akzeptanzwertorientierungen zu Selbstwertorientierungen “5, von Steigerung der psychischen und emotionalen Anforderungen an die Ehe ${ }^{6}$. Außerdem werden äußere Faktoren namhaft gemacht, vor allem die gestiegene Erwerbstätigkeit und ökonomische Unabhängigkeit der Frau, ${ }^{7}$ der ,Wegfall ökonomischer und sozialer Zwänge, die Optionserweiterung als Grundzug der Veränderung der Lebensbedingungen in den letzten Jahrzehnten" ${ }^{8}$ und anderes mehr.

Aus juristischer Sicht fällt auf, dass bei diesen Einschätzungen einerseits der staatlich-rechtliche Begriff der Ehe zugrunde gelegt, andererseits aber bei der Ursachenforschung der rechtliche Hintergrund möglichst ausgeblendet wird.

Zum ersten: Die Heirats- und Scheidungsstatistiken und ihre Erklärungen beziehen sich auf die Ehe des Bürgerlichen Gesetzbuches, eine Ehe, deren Begründung staatlich registriert und die mit vielfältigen Rechtswirkungen ausgestattet wird. Es geht um die „standesamtliche“, die „bürgerliche Ehe“.

Nun ist dieser Ehebegriff - historisch gesehen - keineswegs allgemeingültig, im Gegenteil eine relativ neue Erscheinung, letztlich eine Übernahme aus dem kirchlichen Eherecht der frühen Neuzeit: Der das Paar trauende Priester ist durch den Standesbeamten ersetzt.

Das erleichtert die Verständigung mit den Juristen, wir haben da keine terminologischen Probleme. Die Folge ist freilich, dass die soziologische Deutung der festgestellten Fakten dann auch nur für diesen Ehetyp (,staatlich registrierte Ehe“) relevant ist. Es fällt einiges heraus, zum Beispiel alle Ehen, die nur kirchlich, aber nicht mit staatlicher Wirkung geschlossen sind (das gibt es im Ausland schon lange, bei uns seit 2009). Vor allem aber bleiben diejenigen auf Dauer angelegten Geschlechtsverbindungen unberücksichtigt, die nicht standesamtlich registriert sind und die man dann mit einer Negation als ,nichteheliche Gemeinschaften“ (neutraler: „Kohabitation“) mit der Ehe kontrastiert. Diese Unterscheidung ist offenbar nicht aus der sozialen Realität der Partnerschaften selbst abgeleitet, sondern aus dem Recht übernommen - im tatsächlichen Leben kann man „Ehen“ und „nichteheliche Gemeinschaften“ praktisch oft nicht unterscheiden.

Die Aussagen über den Rückgang der Bedeutung von Ehe, die aus Standesamtsstatisten herausgelesen wird, bezieht sich also auf einen rechtsbegrifflich eingeschränkten Tatbestand. Sie werden in dem Augenblick relativiert, in dem man auf ein transtemporales oder auch transnationales Eheverständnis Bezug nimmt - es gibt ja noch immer Staaten, in denen man „ohne Staat“ heiraten und auch sich „ohne Staat" scheiden kann. So gesehen signalisiert der Rückgang der standesamtlichen Heiratszahlen in Deutschland zunächst nur eine abnehmende Bereitschaft, sich unter das Regime des vom Staat angebotenen rechtlichen Ehemodells zu stellen, vielleicht aber nicht unbedingt die Ablehnung der

5 R. Nave-Herz, Familie heute. Wandel der Familienstrukturen und Folgen für die Erziehung, 5. Aufl. 2012, S. 123; Rupp (wie Fn. 3), S. 46 (Veränderungen des Wertesystems).

6 R. Nave-Herz et al., Scheidungsursachen im Wandel. Eine zeitgeschichtliche Analyse des Anstiegs der Ehescheidungen in der Bundesrepublik Deutschland, Bielefeld 1990, S. 138; Kaufmann (wie Fn.

1), S. 119; Hill/ Kopp (wie Fn. 1), S. 47.

7 Dazu unter anderem: Kaufmann (wie Fn. 1), S. $103 \mathrm{ff}$.

8 Kaufmann (wie Fn. 1), S. 121. 
Ehe (des ehelichen Lebens) in einem substantiellen Sinn. Meinungsbefragungen zeigen, dass die Ehe nur selten abgelehnt wird, man entscheidet sich aber immer seltener dafür.

$\mathrm{Ob}$ darin eine „De-Institutionalisierung“, „Individualisierung“ usw. zum Ausdruck kommt, hängt davon ab, wie sich „Ehe“ und ,nichteheliche Lebensgemeinschaft“ in ihrer sozialen und psychologischen Realität eigentlich unterscheiden. Die These von der „DeInstitutionalisierung“ greift dann, wenn man den soziologischen Institutionenbegriff an die rechtliche Struktur anbindet, aber schon nicht mehr, wenn man „dauerhafte Paarbeziehung“" als solche den sozialen Institutionen zurechnet. ${ }^{9}$

$\mathrm{Zu}$ nichtehelichen Gemeinschaften in Deutschland liegt eine Reihe von aufschlussreichen Untersuchungen vor. Dabei wird der unterschiedliche rechtliche Rahmen gegenüber der Ehe angesprochen, zum Teil auch kritisiert. ${ }^{10}$ Aus rechtspolitischer Sicht ergibt sich folgendes Grundproblem: Das staatliche Eherecht bietet den Paaren - inzwischen auch den homosexuellen - eine rechtliche Struktur für ihre Beziehung an mit erzwingbaren Solidarpflichten auch für den Fall von Trennung und Scheidung, zudem aber mit sehr attraktiven, begünstigenden Rechtswirkungen (gesetzliches Erbrecht, extrem günstiger Satz bei der Erbschaftssteuer, Hinterbliebenenrente, Vorteile bei der Einkommensteuer usw.). Unser ganzes Rechtssystem ist durchzogen von Besonderheiten, die nur für Ehegatten und eingetragene Lebenspartner gelten. Wodurch ist das gerechtfertigt?

Allein durch den Umstand, dass die Ehe behördlich registriert und damit zuverlässig feststellbar ist, die Existenz anderer Gemeinschaften hingegen nicht? Oder setzen die enormen Unterschiede in der rechtlichen Behandlung auch eine substantielle Differenz zwischen den Erscheinungsformen der Paarbeziehung voraus? Und worin könnte sie liegen? Ergeben sie sich aus Unterschieden in Dauer ${ }^{11}$ oder Intensität? ${ }^{12}$ Wenn der Rückgang der Heiratsziffern mit den gestiegenen emotionalen Anforderungen erklärt wird sind also die emotionalen Anforderungen für Kohabitanten geringer? Entlastet die Ehe als rechtsverbindliche Solidargemeinschaft die Gesellschaft mehr als das nichteheliche $\mathrm{Zu}-$ sammenleben? Wie entwickeln sich die Solidarbeziehungen nach einer Trennung? Liegt der Unterschied auch darin, dass die nichteheliche Lebensgemeinschaft begrifflich eine sehr viel variablere Lebenswirklichkeit abdeckt als die Ehe?

Ferner: Welchen Einfluss haben die Rechtsvorstellungen der Beteiligten bei der Entscheidung für Ehe oder eheloses Zusammenleben? Wollen beide oder einer von ihnen nicht heiraten, weil sie das rechtliche Paket ablehnen? Oder einen Teil dieses Pakets? (Das wäre relevant für die Frage, ob wir wie Frankreich eine Art Miniehe - Solidaritätspakt - einführen sollten). Oder sind umgekehrt die rechtlichen Effekte ein tragendes Motiv für den Heiratsentschluss? Inwieweit haben die Paare überhaupt rechtliche Vorstellungen über ihre Art des Zusammenlebens?

9 Burkart (wie Fn. 1), S. 171 („,das konjugale Paar“). Die Zweierbeziehungen sind auch Gegenstand übergreifender Untersuchungen vgl. K. Lenz, Soziologie der Zweierbeziehung, Opladen 1998.

10 Vaskovics/Rupp/Hofmann (wie Fn. 1), S. 25.

11 Die Stabilität von nichtehelichen Partnerschaften wird bei Kaufmann (wie Fn. 1), S. 108, hoch eingeschätzt, andere betonen die geringere Stabilität von nichtehelichen Gemeinschaften, so D. Lois, Lebensstile und Entwicklungspfade nichtehelicher Lebensgemeinschaften - eine empirische Analyse mit dem sozioökonomischen Panel, Wiesbaden 2009, S. 230.

12 Ein Vergleich Ehe - nichteheliche Lebensgemeinschaft findet sich in der Untersuchung von Lois (wie Fn. 11), S. 107f. 
Letztlich läuft das auf die Frage hinaus: Welche Rolle spielen Recht und Rechtsvorstellungen, Gesetzgebung und Rechtsprechung in der soziologischen Analyse von gesellschaftlichen Entwicklungen? Ist es zureichend, das Recht als „Randbedingung“ zu sehen $?^{13}$ Bildet das Recht nur die Begleitmusik oder einen eigenen Faktor, der soziale Bewegungen beschleunigen, behindern, vielleicht auch auslösen kann?

Der Vergleich Ehe-Kohabitation wird - um auch das noch anzufügen - dadurch komplizierter, dass man die bürgerliche Ehe durch Verträge entsolidarisieren (Ausschluss von Vermögens- und Scheidungsfolgen), andererseits das nichteheliche Zusammenleben durch Verträge verrechtlichen kann. Dafür gibt es eine notarielle Praxis ebenso wie Vertragsmuster auf dem Markt. Die Differenz zwischen Ehe und eheloser Gemeinschaft kann also von beiden Seiten her eingeebnet werden, in welchem Ausmaß dies in der Praxis geschieht, wäre zu eruieren.

\section{Ehescheidung}

Auch die Scheidungsforschung ist zumindest überwiegend auf die Ehescheidung im juristischen Sinne konzentriert - also: Auflösung der bürgerlichen Ehe durch Richterspruch.

Das bedeutet: Bei der Erklärung der „Ehescheidung und ihrer Ursachen“ bleiben gewöhnlich die faktischen Trennungen von Ehegatten unberücksichtigt. Allerdings kann die Trennung eine Alternative zur Scheidung sein - man denke an das katholische Ehepaar, das sich aus religiösen Gründen nicht scheidet, wohl aber trennt. Bei Interpretation der Entwicklung der Scheidungsraten müsste in Anschlag kommen, dass nach dem früheren Eherecht zahlreiche Ehen gar nicht geschieden werden konnten, auch wenn ein Partner oder beide dies wollten. Der Ausweg war das permanente Getrenntleben, das in den Scheidungsstatistiken nicht erscheint. ${ }^{1}$

Auf der anderen Seite wird bei der Erforschung der Ursachen für die seit 1979/80 in Deutschland zweifellos stark angestiegenen Scheidungszahlen der rechtliche Rahmen eher beiläufig berücksichtigt. Das ist insofern logisch, als die rechtliche Möglichkeit, sich scheiden lassen, nicht das eigentliche Motiv für Scheidungsentschlüsse bildet. Man scheidet sich nicht allein deshalb, weil es juristisch möglich ist. Doch kann man fragen, ob im Rahmen der Deutung der Scheidungsursachen - nicht auch das jeweils geltende Recht selbst einen Faktor ausmacht.

Seit 1900 galten in Deutschland - im groben Überblick - drei Scheidungsrechte:

- Scheidung nach Verschuldensprinzip nach dem BGB von 1900;

- Scheidung nach Verschuldensprinzip plus eingeschränkter Zerrüttungsscheidung nach dem Ehegesetz von 1938;

- reine Zerrüttungsscheidung ab 1. Juli 1977 - mit der praktisch sicheren Möglichkeit, eine Ehe sogar einseitig auflösen zu lassen. Die Hindernisse, die das neue Recht der Scheidung entgegensetzt, schieben die Scheidung allenfalls hinaus.

13 „Individuelle Randbedingungen hinsichtlich juristischer Barrieren“ bei Hill/Kopp (wie Fn. 1), S. 229.

14 Zutreffend R. Nave-Herz et al., Scheidungsursachen im Wandel. Eine zeitgeschichtliche Analyse des Anstiegs der Ehescheidungen in der Bundesrepublik Deutschland, Bielefeld 1990, S. 46. 
Betrachtet man die Scheidungsraten (also Scheidungszahlen im Verhältnis zur Zahl der Eheschließungen) auf dem Hintergrund dieser Scheidungsrechte, so ergibt sich für die Phase des ersten Scheidungsrechts (Verschuldensscheidung) eine Rate von unter 10\%, für die Phase des zweiten Scheidungsrechts (Verschulden und Zerrüttung) eine Rate zwischen 10 und 20\%, für die Phase des dritten Scheidungsrechts (reine Zerrüttung) von 40 bis $50 \%{ }^{15}$

Dabei habe ich die Kriegszeiten ausgeblendet, ebenso die Tatsache, dass die Scheidungszahlen kurz vor der Reform von 1977 außergewöhnlich nach oben schnellten, in den ersten Jahren nach der Reform zunächst nach unten sackten. Nach einer gewissen Anlaufzeit in den ersten Jahren nach der Reform betrug die Scheidungsrate 1980 bereits 28,4\%, erreichte ihren bisherigen Kulminationspunkt 2003 mit 55,8\% und bewegt sich seitdem leicht rückläufig zwischen 40 und 50\% (2012: 46,27). ${ }^{16}$ Also: Drei Scheidungsrechte, drei extrem unterschiedliche Scheidungsquoten.

Auch das starke Ansteigen der Scheidungszahlen in den Jahren vor der Reform 1977 (z.B. Scheidungsrate im Jahre $1976=29,9 \%$ ) lässt sich in einen Bezug zu den Rechtstatsachen bringen. Die Scheidungsrechtsreform war in den Jahren vor ihrem Inkrafttreten in der Öffentlichkeit stark diskutiert worden, je näher sie rückte, desto mehr versuchten Partner einer unglücklichen Ehe, noch schnell das alte Scheidungsrecht zu nutzen, um nicht den Scheidungsfolgen des neuen ausgesetzt zu sein (Unterhaltspflicht ohne Rücksicht auf Verschulden, Rententeilung), die Anwälte wurden mit Scheidungswünschen überrannt. Und ebenso ist erklärbar, dass in den ersten beiden Jahren nach der Reform 1977 die Scheidungsraten erst einmal absackten - vieles, was zu scheiden war, war eben noch schnell nach altem Recht erledigt worden, das neue Recht war noch unerprobt und in seinen Konsequenzen unsicher. ${ }^{17}$

Nun sind die Scheidungsquoten ein sehr grobes Raster. Es soll nicht gesagt sein, dass die Entwicklung der Scheidungsraten einfach nur ein Effekt der jeweiligen Scheidungsrechte ist; das verbietet sich schon im Hinblick darauf, dass der Rückgang der EheschlieBungen sich auf die Scheidungsquote auswirkt. Die dauerhafte Zuordnung extrem unterschiedlicher Scheidungsquoten zu den Zeiten unterschiedlicher Scheidungsrechte frappiert aber doch. Die Frage aber ist, ob die vielfältigen Erklärungen für die Ursachen zu relativieren sind, wenn man das Recht als Einflussfaktor in Betracht zieht.

Nun kann man sagen: Das Recht kommt nicht vom Himmel, sondern aus den Köpfen von Abgeordneten und Ministerialbeamten, die ihrerseits unter dem Einfluss der öffentlichen Meinung stehen. Auch wenn das Recht ein Einflussfaktor ist, gehört es eben zur gesellschaftlichen Entwicklung im Ganzen. Der Vorgang, den wir als „De-Institutionalisierung“, „Individualisierung“ etc. deuten, findet dann zuerst im gesellschaftlichen Bewusst-

15 Entsprechenden Proportionen ergeben sich auch, wenn man die Zahl der gerichtlichen Ehelösungen mit der Zahl der bestehenden Ehen oder mit der Zahl der Einwohner in Verhältnis setzt, vgl. die Tabelle bei Nave-Herz et al. (Fn. 14), S. 13f.

16 Diese auf- und abgerundeten Werte sind errechnet nach den Angaben über Eheschließungen und Ehescheidungen des Statistischen Jahrbuches für das Deutsche Reich und des Statistischen Bundesamts.

17 Unzureichend ist die bei Kaufmann (wie Fn. 1), S. 118 referierte Auffassung, der scharfe Einschnitt der Scheidungshäufigkeit in den Jahren 1978/79 sei auf die Verlängerung des Scheidungsverfahrens zurückzuführen. 
sein der rechtspolitischen Führungsschichten statt, das sich dann à la longue in Gesetzen niederschlägt und so wiederum das praktischen Handeln der Menschen beeinflusst.

Diese Zusammenhänge - wenn man sie so nimmt - würden aber nicht hindern, der Entwicklung von Recht und Rechtsvorstellung ein eigenes Gewicht in der Deutung der gesellschaftlichen Entwicklungen zuzumessen. Es sind ja oft auch nur Zufälle, die das Zustandekommen eines Gesetzes ermöglichen oder verhindern und nicht immer trifft die Gesetzgebung auch die tatsächliche gesellschaftliche Situation. Wenn das Recht - als bewusste, gezielte Normsetzung - ein mitbestimmender Faktor ist, dann wird die Vorstellung von einer durch autonome Verhaltensänderungen (psychologische Trends etc.) allein erklärbaren gesellschaftlichen Entwicklung zweifelhaft. Gesellschaftliche Entwicklung fließt dann nicht einfach dahin, sondern wird zu einem gewissen Anteil ,gemacht“.

Der Austausch des Rechts kann sogar auch Zweifel daran wecken, ob die ,Scheidung des Jahres 1900“ das Gleiche ist wie die „Scheidung im Jahre 2014“, zumal wenn man das völlig veränderte Scheidungsfolgenrecht hinzunimmt. Kaufmann vermutet eine kulturelle Umdeutung der Ehescheidung. ${ }^{18}$ Natürlich ändert das nichts an der Rechtsnatur der Scheidung als Auflösung des rechtlichen Ehebandes, aber möglicherweise hat die Scheidung Funktionen übernommen, die vorher durch Trennung geleistet wurden.

\section{Kinder und Eltern}

Die Frage der Bedeutung des Rechts für die Interpretation gesellschaftlicher Entwicklungen scheint besonders aktuell, wenn es um das Verhältnis von Kindern und Eltern geht. Elternschaft und Kindschaft sind in verschiedener Hinsicht vom Recht festgelegt, angefangen bei der Zurechnung von Elternschaft bis hin zur Zuordnung des Sorgerechts oder von begrenzten Rechtspositionen wie Umgang, Auskunftsrechten oder Unterhaltsverpflichtungen.

Offenkundig gehen diese rechtlichen Zuschreibungen in soziologische Erhebungen und Analysen nur partiell ein. Hauptsächlich finden wir eine soziologische Terminologie vor, die mit der juristischen geradezu kontrastiert ${ }^{19}$ (also anders als bei Ehe und Ehescheidung). Die Untersuchungen zur Kindschaft orientieren ihre Unterscheidungen meist daran, bei wem das Kind alltäglich lebt, in wessen Haushalt es sich befindet. Daran knüpft die Differenzierung zwischen „Elternfamilie“ und „Ein-Eltern“-Familie bzw. „Mutterfamilie“ und „Vaterfamilie“ an. ${ }^{20}$ Die „Ein-Eltern-Familie“ wird auch mit dem Terminus „alleinerziehender Elternteil“" verbunden. Dieser erfreut sich einer besonderen Aufmerksamkeit der Forschung (Häufigkeit des Vorkommens, Ursachen der Ein-Elternschaft, Befindlichkeit der Kinder, Lebensprobleme der Eltern).

18 Kaufmann (wie Fn. 1), S. 119.

19 Grundlegend L. A. Vaskovics, Segmentierung und Multiplikation von Elternschaft. Konzept zur Analyse zu Elternschafts- und Elternkonstellationen, in: D. Schwab/L. A. Vaskovics, Pluralisierung von Elternschaft und Kindschaft. Familienrecht, -soziologie und -psychologie im Dialog. Sonderheft 8 der Zeitschrift für Familienforschung/Journal of Family Research, Opladen/Farmington Hills, S. 11, 13, 17.

20 Diese Begriffe z.B. bei Nave-Herz (wie Fn. 5), S. 95 ff. 
Der Begriff ,„alleinerziehend“ wird allerdings unterschiedlich gesetzt:

- Einige Autoren meinen die Konstellation, dass nur ein Elternteil - Mutter oder Vater - mit den Kindern zusammenlebt und die alltägliche Erziehungsverantwortung allein wahrnimmt. ${ }^{21}$ Diese Definition schließt die Fälle ein, in denen im Haushalt außerdem noch ein weiterer Erwachsener vorhanden ist, z.B. der neue Partner der kindesbetreuenden Mutter. Nach dieser Terminologie ist beispielsweise die Mutter, die nach der Scheidung mit den Kindern zu ihrem Freund gezogen ist, ,alleinerziehend“. Von nebensächlicher Bedeutung - so wird gesagt $-{ }^{22}$ sei die Sorgerechtsregelung.

- Andere Darstellungen wie neuerdings auch das Statistisches Bundesamt beschränken den Begriff ,alleinerziehend“ auf die Fälle, in denen der betreuende Elternteil als alleiniger Erwachsener mit Kind in einem Haushalt lebt ${ }^{23}$ (volljährige Geschwister des Kindes werden hier nicht als Erwachsene gerechnet). Die Stieffamilie (neuer Ehepartner im Haushalt) oder Quasi-Stieffamilie (neuer Lebensgefährte im Haushalt) lässt die Einordnung unter die „Alleinerziehenden“ entfallen. Dabei scheint die Vorstellung maßgeblich, dass das ständige Zusammenleben eines neuen Lebenspartners mit einem Elternteil und dessen Kind in aller Regel faktisch zu einer Übernahme der Elternrolle - zur Miterziehung - führt.

Diese doppelte Bedeutung des soziologischen Begriffs ,alleinerziehend“ kann zu Unklarheiten führen (manchmal wird auch von ,alleinerziehend“ im engeren und im weiteren Sinne unterschieden). ${ }^{24}$

So oder so entspricht die Terminologie nicht den Rechtsbegriffen. „Erziehung“ im Rechtssinn ist keineswegs identisch mit dem Leben im selben Haushalt. Die Termini „Pflege und Erziehung“ umschreiben das umfassende, rechtlich eingerichtete Sorgerechtsverhältnis zwischen Kind und Eltern oder zwischen Kind und Personen, die an die Stelle der Eltern getreten sind. Zwar verwirklicht sich die Elternsorge meist auch im häuslichen Zusammenleben mit ihm, aber nicht nur und nicht notwendigerweise. Die gemeinsame Pflege und Erziehung des Kindes ist auch das rechtliche Ideal auch für den Fall, dass die Eltern sich trennen oder scheiden, das Kind aber alltäglich in der Obhut nur eines Teils lebt; juristisch gesehen bleibt der andere „miterziehend“.

Dem soziologischen Tatbestand „alleinerziehend“ - sei es im engeren wie im weiteren Sinne - können ganz unterschiedliche Rechtslagen zugrunde liegen. Man kann sogar ein Kind faktisch allein betreuen (,allein erziehen“ im soziologischen Sinne), ohne Inha-

21 Nave-Herz (wie Fn. 5), S. 95: ,jene Familien, in denen ein Elternteil (Mutter oder Vater) die alltägliche Erziehungsverantwortung für ein Kind (bzw. für mehrere) besitzt, mit dem es in einer Haushaltsgemeinschaft wohnt." Letztlich unklar F. Nestmann/S. Stieler, Wie allein sind Alleinerziehende? Soziale Beziehungen alleinerziehender Frauen und Männer in Ost und West, Opladen 1998, S. 12 (Einelternfamilie als „Haushaltsgemeinschaft von mindestens zwei Personen ..., von denen nur eine die erwachsene Bezugsperson ist und die anderen minderjährige Kinder sind").

22 Nave-Herz (wie Fn. 5), S. 95.

23 Statistisches Bundesamt, Datenreport 2013, S. 46; ebenso Jahrbuch 2013: „Alleinerziehende: Mütter und Väter, die ohne Ehe- oder Lebenspartnerin bzw. Lebenspartner mit ihren minder- oder volljährigen Kindern in einem Haushalt zusammenleben.“ Dem folgend z.B. D. Lois/J. Kopp, Elternschaftskonstellationen bei Alleinerziehenden, in: Schwab/Vaskovics (wie Fn. 19), S. 59, 60; N. F. Schneider et al., Alleinerziehen - Vielfalt und Dynamik einer Lebensform, Stuttgart 2001.

24 Nestmann/Stieler (wie Fn. 21), S. 53. 
ber der elterlichen Sorge zu sein. So ist es z.B. bei Pflegeeltern, denen vom Gesetz nur sehr beschränkte Sorgebefugnisse eingeräumt werden, während das Sorgerecht selbst bei den Eltern bleibt. Umgekehrt kann man das alleinige Sorgerecht innehaben, ohne überhaupt das Kind faktisch zu erziehen, indem man es in ein Heim gibt.

Die Sorgerechtslagen können sich sehr kompliziert gestalten. Es gibt Fälle, in denen das Sorgerecht geteilt ist. Schon das normale gemeinsame Sorgerecht nach Trennung der Eltern ist gewöhnlich aufgespaltet: bei in Angelegenheiten von erheblicher Bedeutung sind beide Teile zuständig, in Angelegenheiten des täglichen Lebens allein der obhutführende Elternteil. Teilungen des Sorgerechts sind auch in anderer Weise möglich. Eine Mischform bietet das heute viel diskutierte Wechselmodell, bei dem das Kind zeitweise bei dem einen und dem anderen Elternteil lebt, wo sich die Eltern sozusagen als jeweils „alleinerziehende“ im soziologischen Sinn abwechseln.

Bei dieser rechtlichen Vielfalt ist es verständlich, wenn die soziologische Forschung die rechtlichen Differenzierungen zunächst einmal vernachlässigt: Das elterliche Sorgerecht ist eine juristische Konstruktion, keine Realität. Die Realität sind das Zusammenleben und die persönliche Begegnung des Kindes mit den Eltern oder mit einem Elternteil auf welcher rechtlichen Basis auch immer. Die Sozialisation des Kindes gelingt aufgrund von tatsächlichen Interaktionen, nicht aufgrund von Rechtslagen. Und deswegen werden die Lebensverhältnisse der Kinder so in den Blick genommen, wie sie sich realiter darstellen.

Aus der Sicht der Juristen ist das begreiflich, erfüllt aber nicht alle Wünsche, weil wir zu gerne wüssten, ob die juristischen Konstruktionen - gemeinsame Sorge bei Trennung, alleinige Sorge bei Trennung, gespaltene Sorge bei Trennung - wirklich nur Schemen sind, juristische Träume, oder doch auch irgendwie auf die Realität durchschlagen. Will man das erforschen, so muss man sich logischerweise auf die unterschiedlichen Rechtslagen einlassen. Auch der Befund „Für das Leben des Kindes ergibt sich kein signifikanter Unterschied unter den verschiedenen rechtlichen Konstrukten“" wäre ja ein interessantes Ergebnis für die Rechtspraxis.

Die Diskrepanz zwischen soziologischer Kategorisierung und rechtlicher Einordnung können wir beim Phänomen „sozialen Elternschaft ${ }^{\text {‘25 }}$ besonders gut beobachten. „Soziale Elternschaft" meint im Gemeingebrauch wie meist auch in der sozialwissenschaftlichen Literatur ein psycho-soziales, tatsächlich gelebtes Eltern-Kind-Verhältnis. Oft gerät noch ein reduzierendes Element hinzu: dann wird der Begriff auf eine Elternschaft bezogen, die nur als psycho-soziale Wirklichkeit besteht, nicht als rechtliche. ${ }^{26}$ Die soziale Elternschaft kann so zum Gegenbegriff der rechtlichen werden, mit dem wenig schönen Ergebnis, dass rechtliche Eltern auch dann, wenn sie hingebungsvoll für ihre Kinder sorgen, nicht als soziale Eltern angesprochen werden (soziale Elternschaft als „nur soziale“ Elternschaft).

Wie dem auch sei, ,soziale Elternschaft" ist kein Terminus des Rechts, das mit ihr Gemeinte ist auch nicht Gegenstand einer spezifischen rechtlichen Regelung. Soziale Elternschaft kann sich auf unterschiedlichem rechtlichen Hintergrund bilden: als Stiefeltern-

$25 \mathrm{Zu}$ diesem Begriff Vaskovics, in: Schwab/Vaskovics (wie Fn.17), S. 15 (für die soziale Elternschaft ist die alltägliche Wahrnehmung der in der Elternrolle enthaltenen normativen Pflichten und Rechte gegenüber dem Kind, dessen Existenzerhaltung und Erziehung konstitutiv).

26 M. Feldhaus/J. Huinink, Multiple Elternschaften in Deutschland, in: Schwab/Vaskovics (wie Fn. 17), S. 77, 79. 
schaft, als Pflegeelternschaft, als Elternschaft einer Heimerzieherin, oder auch ohne Rechtsbeziehung, z.B. im Rahmen einer nichtehelichen Gemeinschaft mit dem Kind des Partners. So wichtig es für das Kind ist, dass es in einer bestimmten Person psychisch und sozial einen Elternteil gefunden hat, so wenig versucht das Recht, diesen Tatbestand generell begrifflich einzufangen und mit Rechtswirkungen auszustatten.

Freilich: Wo immer eine staatliche Entscheidung (Behörde, Gericht) sich nach dem Kindeswohl auszurichten hat, sind die persönlichen Bindungen des Kindes von Bedeutung, Bindungen an Großeltern, Geschwister, Verwandte, Erzieherinnen, Lebensgefährten der Mutter usw. Das Recht scheut sich indes, den Begriff der sozialen Elternschaft zu verwenden. Das erklärt sich daraus, dass die genannten Bindungen von ganz unterschiedlicher Art und Dichte sein können. Man möchte den „rechtlichen Eltern“ auch nicht auf derselben sprachlichen Ebene („Eltern“) einen Gegenpol gegenüberstellen. Denn meist sind bei der faktischen Elternschaft einer Bezugsperson die rechtlichen Eltern nach wie vor vorhanden, kümmern sich möglicherweise ihrerseits um das Kind, etwa bei der Familienpflege, wenn die Herkunftseltern den Kontakt halten und Unterhalt leisten. Man will das Bild von verschiedenartigen mit einander konkurrierenden Eltern vermeiden. Immer lässt sich die mehrfache Elternschaft freilich nicht vermeiden, etwa wenn leibliche und rechtliche Elternschaft auseinanderfallen, doch seien diese Fälle hier ausgeblendet.

Um die Realität einfangen, behilft sich das deutsche Recht mit dem vom Bundesverfassungsgericht erfundenen Begriff der „,sozial-familiären Beziehung“. ${ }^{27}$ Eine solche wird unter folgenden Voraussetzungen angenommen: 1) Es muss sich um eine enge Bezugsperson des Kindes handeln, 2) sie muss für das Kind tatsächliche Verantwortung tragen oder getragen haben. Dabei ist eine Übernahme tatsächlicher Verantwortung in der Regel anzunehmen, wenn die Person mit dem Kind längere Zeit in häuslicher Gemeinschaft zusammengelebt hat. Hier orientiert sich das Recht also wie auch die Soziologie an dem Tatbestand des Lebens im selben Haushalt als Indikator für eine persönliche Zuwendung. Eine elterngleiche Rolle wird nicht verlangt - sie wäre auch schwer überprüfbar. Aber immerhin! Das Recht will den Fakten Rechnung tragen.

Nun muss man allerdings sehen, dass die Rechtsordnung die sozial-familiäre Beziehung keineswegs zu einem elternähnlichen Institut ausbaut, sondern nur sehr partikulare Wirkungen damit verbindet, vor allem ein Umgangsrecht mit dem Kind, das gerade dann relevant wird, wenn die Haushaltsgemeinschaft schon aufgelöst ist, die soziale Elternschaft also möglicherweise gar nicht mehr besteht. Von Sorgerecht ist nicht die Rede, auch nicht für die Zeit des Zusammenlebens: Der Lebensgefährtin des sorgeberechtigten Vaters z.B. ist in die Erziehung soweit einbezogen als der Vater dies will, ihre „soziale Elternschaft" ist in jederzeit widerrufbarer Weise nur abgeleitet.

Dass die soziale Elternschaft nicht auch als Rechtsfigur erscheint, mag die Soziologie enttäuschen. Sie hat ein soziales Gebilde vor Augen, eine gelebte Familie, die aber vom Recht nicht als Einheit erfasst, geschweige denn als Einheit geregelt wird. Die „sozialfamiliäre Beziehung“ der Juristen andererseits ist als soziologischer Forschungsgegenstand schwierig, weil diffus: Sozialfamiliäre Bezugsperson kann der zeitweilige Lebenspartner eines Elternteils ebenso sein wie die Tante, bei der das Kind einen Teil des Tages

$27 \mathrm{Zu}$ Begriff und rechtlicher Bedeutung M. Lipp, Elternschaft, „sozial-familiäre Beziehung“ und „Bindungsperson“, in: Schwab/Vaskovics (wie Fn. 17), S. 121ff.; Schwab (ebenda), S. 51. 
verbringt oder der Erzieher im Internat. Weil die Rechtsfolgen diffus sind, ist es auch der Tatbestand, denn was heißt schon ,tatsächliche Verantwortung für das Kind tragen“ Verantwortung wofür genau, in welchem Umfang, in welcher Weise?

Der Kontrast zwischen Recht und sozialem Leben kulminiert bei der sogenannten Patchworkfamilie. Diese erscheint in den Sozialwissenschaften als ein besonderer Familientypus, dessen gehäuftes Aufkommen nach der Vorstellung Vieler die so genannte traditionelle Familie (genetische Eltern = rechtliche Eltern = soziale Eltern) abzulösen begonnen hat.

Die Juristen kennen zwar den Begriff „Patchwork-Familie“ und übernehmen die soziologische Deutung als Indikator des familiären Wandels. Im Regelwerk des Rechts findet sich die Patchworkfamilie weder unter diesem noch unter einem anderen Terminus. Juristisch wird sie in eine Vielzahl von einzelnen Familienbeziehungen zerlegt: Eltern-Kind, Stiefeltern-Stiefkind, Geschwisterbeziehung und so weiter. Die Summe der Rechtsverhältnisse macht noch nicht einmal das Ganze aus. So bleibt z.B. die Beziehung unter den Kindern, die jeder Partner aus früheren Verbindungen in die Patchworkfamilie einbringt, juristisch unbenannt, es gibt keine „Stiefgeschwisterschaft“ im rechtlichen Sinne.

Das stößt möglicherweise auf wenig Verständnis in den Sozialwissenschaften. Man könnte denken: Dem sozialen Tatbestand einer familiären Einheit muss auch das Recht eine Struktur geben. Das ist ein Punkt, in dem die Wissenschaften in einen Diskurs miteinander kommen sollten. Vor rechtlicher Seite könnte man sagen. Es ist nicht die Aufgabe des Rechts, die soziale Wirklichkeit abzubilden, sondern für die - ja oft genug sehr komplizierten - Verhältnisse geeignete Konfliktregeln zur Verfügung zu stellen. Die Patchworkfamilie steht nicht für sich allein, sondern im Schnittpunkt von familienrechtlichen Bezügen auch zu außenstehenden Personen, dem anderen Elternteil und dessen Eltern usw. Die Patchworkfamilie ist - juristisch gesehen - eine Koinzidenz von mehreren Familien.

Doch lässt sich fragen: Kann es sein, dass die rechtliche Aufspaltung einer sozialen Familie in verschiedene Individualverhältnisse das Ganze aus dem Blick verliert und wichtigen Regelungsbedarf vernachlässigt? Beispielsweise: Muss nicht auch der nichteheliche Partner des erziehenden Elternteils in seiner Rolle als Stiefelternteil und als ein Angehöriger im Rechtssinne verstanden und anerkannt werden?

Mit Fug gewinnen die Soziologen aus ihrer Sicht der Dinge ein Potenzial für rechtspolitische Fragestellung und Kritik. Die soziologische Analyse selbst geht nicht selten über Beschreibung und Deutung hinaus und wagt den Sprung in die Normativität, in das „Sollen“. ${ }^{28}$ Die Frage ist dann, aus welcher Quelle dieses „Sollen“ gespeist wird. Ist es die bloße Anschauung der Faktizität („Patchworkfamilie als reale Erscheinung“) oder doch eine normative Grundlage: Kindeswohl, widerspruchsfreie Regelung der Verantwortung für ein Kind, Verantwortung der Gesellschaft für Kinder und Familien. Beim Sprung der Soziologie in die Rechtspolitik finden sich unsere Wissenschaften auf demselben Feld wieder - umso dringender der gegenseitige Austausch.

28 Z.B. Vaskovics, in: Schwab/Vaskovics (wie Fn. 1), S. 11, 33ff.; W. Lauterbach, Bedeutung der Abstammung für die Familien- und Verwandtschaftszugehörigkeit, ebenda, S. 205f.; Feldhaus/Huinink, ebenda, S. 77, 100f.; Hill/Kopp (wie Fn. 1), S. 230. 


\section{Resumee}

1) Sozial- und Rechtswissenschaften kommen auf dem Feld der Familienforschung ohne einander nicht aus. Die rechtlichen Befunde können die auf die Fragestellungen der Sozialwissenschaften Einfluss haben, die Ergebnisse der soziologischen Forschung schaffen die Basis für kritische Distanz zum jeweils geltenden Recht.

2) Voraussetzung einer fruchtbaren Zusammenarbeit ist die Offenlegung der unterschiedlichen Grundverständnisse, Sichtweisen, Methodik und Terminologie.

3) Für die Soziologie stellt sich die Frage, inwieweit die jeweils gegebenen Rechtslagen als Entwicklungsfaktoren in die Interpretation der gesellschaftlichen Realität Eingang finden können oder sollen.

4) Für die Rechtswissenschaft stellt sich die Frage, ob sie bei der Entwicklung von normativen Konzepten sich der Hilfe der Sozialwissenschaften im erforderlichen Umfang versichert, namentlich: inwieweit Realitätsvorstellungen, die ihren Projekten zugrunde liegen, sozialwissenschaftlich hinreichend abgesichert sind.

5) Für beide Wissenschaftszweige stellt sich die Frage, inwieweit die Beschreibung von Realität mit Begriffen geschieht, die offen oder subkutan bereits normative Elemente oder Wertungen enthalten, welche die Tendenzen des Begriffsgebrauchs vorentscheiden.

Wir sollten also zusammenarbeiten, nicht nur interdisziplinär, sondern, wenn es denn geht, transdisziplinär, um ein in Mode gekommenes Wort aufzugreifen.

Eingereicht am/Submitted on: 15.10.2015

Angenommen am/Accepted on: 05.04.2015

Anschrift des Autors/Address of the author:

Prof. Dr. jur. Dr. h.c. Dieter Schwab

Universität Regensburg

Lehrstuhl für Bürgerliches Recht, Internationales Privatrecht

und Rechtsvergleichung (Prof. Dutta)

Universitätsstraße 31

93053 Regensburg

Deutschland/Germany

E-Mail: dieter.schwab@ur.de 